\section{Artículo de reflexión}

Cómo citar: Conrado, Y., y Henao, L. (2020). Un abordaje teórico de la experiencia emocional del ser maestro. Praxis Pedagógica, 20(26), 76-94. https://doi.org/10.26620/ uniminuto.praxis.20.26.2020.76-94

ISSN: 0124-1494

eISSN: $2590-8200$

Editorial: Corporación Universitaria Minuto de Dios - UNIMINUTO

Recibido: 20 de octubre de 2019 Aceptado: 28 de diciembre de 2019 Publicado: 5 de febrero de 2020

Conflicto de intereses: los autores han declarado que no existen intereses en competencia.

\title{
Un abordaje teórico de la experiencia emocional del ser maestro ${ }^{1}$
}

\author{
A theoretical approach to the \\ teachers' emotional experience
}

\author{
Uma abordagem teórica da \\ experiência emocional da \\ profissão do professor
}

\section{Resumen}

Este artículo de reflexión tiene por objetivo mostrar que las emociones no son solo reacciones ante lo que rodea al ser humano, en este caso al maestro, sino que son la respuesta a un estímulo que se genera de forma consciente o inconsciente, y que y que se visibiliza en actividades del diario vivir a partir de la interacción con los otros, y que son mediadas por la tradición y la cultura, que en ocasiones desconoce el carácter necesario de cada una de estas.

Palabras clave: emociones, maestro, experiencia.

\begin{abstract}
This reflexive paper pretend to show how emotions are not only reactions towards that surround the human being, in this case, the teacher, but are also a response to a stimulus, who can occur consciously or unconsciously and can be seen in daily activities when they interact with other beings. On the other hand, tradition and culture mediated emotions, but sometimes are unaware of the necessary character of emotions.
\end{abstract}

Keywords: emotions, teachers, exprierence.

lizmariana10@gmail.com Magíster en Psicopedagogía Vermont School Medellín Colombia

Yuly Natalia Conrado M. Magíster en Psicopedagogía Secretaría de Educación Colombia
1 El presente artículo de reflexión es producto de la investigación: Resignificación del ser maestro: una mirada psicopedagógica a su experiencia emocional, realizada por las autoras para optar al título de Magíster en Psicopedagogía. 


\section{Resumo}

Este artigo procura, por meio duma reflexão sobre a profissão do professor, mostrar que as emoções não só são reações diante do que rodeia ao ser humano, senão que são uma resposta para um estímulo que gera-se de maneira consciente ou inconsciente. Estas são mais notáveis em atividades diárias na interação com colegas, estudantes e pais de família, além de ser mediadas pela tradição e a cultura, as quais, em alguns momentos, desconhecem o caráter necessário de cada uma destas.

Palavras-chave: experiência, professor, emoções e psicopedagogía.

\section{Introducción}

Las emociones son aquellas que movilizan la acción humana, "conforman el paisaje de nuestra vida mental y social" (Nussbaum, 2011, p. 21) y se configuran en la interacción con el otro, es decir, en el contexto sociocultural. Históricamente se han desarrollado diversas concepciones sobre las emociones, incluso se les ha llegado a considerar perjudiciales para los seres humanos, en especial, para los maestros, a quienes se les ha llevado a dejarlas fuera del aula.

De acuerdo con lo anterior, en la primera parte del artículo se hablará del ser maestro desde la intención con la que fue creada la profesión y los retos que enfrenta actualmente en relación con su experiencia emocional. Para ello se toman como referente a autores como Zuluaga, Abramowski, Sánchez Tortosa, entre otros. En el segundo apartado se intentan develar aquellas emociones de las que poco se habla y que permean el acto educativo, como la ira, el miedo, la empatía, la vergüenza y la repugnancia, desde los aportes teóricos de autores como Nussbaum, Abramowski, Camps, entre otros. Para terminar, se presentan algunas conclusiones.

\section{El ser maestro y su experiencia emocional}

En el siglo XV se plantea la necesidad de un sujeto que oriente el aprendizaje de las generaciones jóvenes, a partir de las doctrinas estatales de la época, las cuales demandaban ciudadanos obedientes, disciplinados, dóciles y productivos; 
por ello, quien dirigiera su educación, debía ser el modelo de todos estos valores, para de esta manera, transmitir las intenciones educativas, es decir, "el maestro debía formar ciudadanos que obedecieran en primer lugar al Estado y en segundo lugar a la Iglesia" (Zuluaga, 1999, p. 40), lo cual permitía conservar el orden social establecido.

De acuerdo con lo anterior, el maestro es visto desde una perspectiva en la que prima la enseñanza objetiva, lo más importante será la relación que teje con sus estudiantes, el cumplimiento de objetivos planteados y los resultados académicos, sin embargo descuida la subjetividad de los implicados en la relación pedagógica y establece que las emociones es algo que debe ser controlado por parte del maestro, pues va en contra de la tan anhelada objetividad. Por este motivo, la práctica pedagógica se limita a las disposiciones de las políticas públicas, las cuales son "una línea de conducta intencional y duradera producida como respuesta a la identificación de un problema de una jurisdicción nacional, regional o local, cuya importancia ha sido percibida y evaluada" (Camilloni, 2011, p.13).

Por tanto, las políticas públicas se incorporan como resultado de una forma de entender la realidad circundante, y en el caso educativo, el maestro suele ser excluido de las discusiones teóricas y legislativas, y es relegado netamente a la enseñanza, lo que pone en evidencia que los entes gubernamentales suelen considerarlo desde una lógica instrumental, lo cual también se entrevé en la perspectiva pedagógica clásica que fue por muchos años hegemónica, y continúa siéndolo en algunos entornos escolares, en los que se intenta reducir al maestro exclusivamente al aula de clase, se inhibe de su subjetividad para dedicarse de forma exclusiva a transmitir conocimientos objetivos.

Esta mirada podría explicar por qué las subjetividades de los maestros han sido poco trabajadas en las investigaciones y propuestas de intervención en el ámbito educativo, pese a las nuevas concepciones que los identifican como seres integrales. En este sentido, la institucionalización cumple un papel importante en este despojo hacia el maestro, ya que establece modelos y normas, a tipo de libreto, que altera su discurso sobre su saber específico, lo lleva a actuar alejado de su ser y le asigna un rol para que desempeñe en la sociedad. 
Así, la educación se convierte en el medio utilizado por las políticas gubernamentales para reproducir su discurso de formación del hombre de acuerdo con el tiempo, la cultura y el contexto; la enseñanza se concibe como un "acontecimiento cultural y de saber" (Zuluaga, 1999, p. 23), pasa a ser el método para alcanzar ese modelo de hombre y de escuela, el espacio donde tiene lugar lo que limita la relación entre el maestro y el saber.

Por esta concepción socio-política clásica, actualmente el maestro debe hacerle frente a algunos retos que afectan su ser emocional, como lo son: en primer lugar, la limitación de la pedagogía a un saber instrumental y la expansión de la visión reduccionista del maestro; en segundo lugar, su subordinación al discurso de las ciencias sociales, que inhibe la expresión de su saber pedagógico, y es la psicología la más común en estos casos, lo que justifica, además, la mirada instrumental que se tiene del educador, y en tercer lugar, su subordinación a las reglas que rigen las instituciones educativas y que desconocen su vinculación al discurso pedagógico.

El primer reto al cual se enfrenta el maestro en su ejercicio profesional es la reducción de la pedagogía a un saber instrumental en las instituciones educativas, desconoce su historicidad y deja de lado su función formadora donde se consideran las diferentes dimensiones del ser humano, para ser entendida desde el tecnicismo de la acción educativa. Para Zuluaga (1999), la pedagogía es "una disciplina que conceptualiza, aplica y experimenta los conocimientos referentes a la enseñanza de los saberes específicos en las diferentes culturas" (p. 11), además, es la encargada de la reflexión constante sobre el discurso y las prácticas pedagógicas.

Así mismo, Bernstein (1988, citado por Díaz, 1995) concibe el campo pedagógico como un espacio estructurado por el contexto de reproducción discursiva, en el que los agentes que hacen parte, sus prácticas y sus posiciones reproducen el discurso pedagógico. (p. 12).

En el afán por restablecer el discurso legítimo sobre la pedagogía, se establecen tensiones entre los grupos de intelectuales, académicos y profesionales que se enfrentan entre sí por la autonomía discursiva o por la hegemonía, estatus y poder, es decir, por delimitar el espacio discursivo y sobre esto fijar criterios de pertenencia o exclusión. 
Esta pugna entre las diversas disciplinas de las ciencias sociales por el control discursivo de la pedagogía, expulsa al maestro y a su saber de la enseñanza, pues no pretende que enseñe desde su subjetividad y ayude a sus estudiantes a construir sus propias subjetividades, sino que busca que solamente se dé una transmisión de contenidos pensados desde el Ministerio de Educación, y que sea un ejecutor de una política pública de determinado gobierno, y le otorga además, a otras disciplinas ajenas a la pedagogía, reflexiones educativas y metodológicas, propias y características de los maestros.

El segundo reto es la subordinación de la pedagogía al discurso de las ciencias sociales, especialmente por la psicología, la cual desplaza el saber pedagógico al ingresar al ámbito educativo, impulsa el conocimiento del sujeto que aprende, trata de apuntar al desarrollo de la infancia y descentra la práctica pedagógica del docente (McWilliam, 1999, citado por Abramowski, 2010, p. 90). Lo "bueno" (lo psicológico) ahora será lo importante en la acción pedagógica.

Es así, como desde hace unas décadas y de manera sostenida, el campo pedagógico se encuentra teñido de psicología, lo que ha dado lugar a que se hable de una "psicologización" de la pedagogía en el ámbito escolar. Se introducen técnicas psicométricas de diagnóstico que permiten identificar diferencias individuales, y a la vez trazan una frontera entre la normalidad y la patología, eleva el discurso patológico del comportamiento, el cual, aunado a la desinstitucionalización actual, pone en entredicho el discurso y la práctica pedagógica, y lleva a los maestros a convertirse, según Varela (1991), en profesionales polivalentes, flexibles y aplicables, pues intenta volverlos tutores, animadores pedagógicos y orientadores, que permanezcan en contacto con las familias y los estudiantes, de forma cálida y afectuosa sin que tengan lugar las emociones y la subjetividad del maestro.

El tercer reto al que se enfrenta el maestro, que dificulta la visibilización de su experiencia integral y limita su vinculación al discurso pedagógico, es la subordinación a las reglas que rigen en cada institución educativa y regulan su decir y su actuar. Es común encontrar en el discurso educativo frases como docente decente, que ponen el comportamiento del profesor en tela de juicio: medido y descalificado, quien debe actuar como un personaje "sin tacha" (Sánchez, 2010, p. 99). De esta manera, podrá establecer límites y normas a 
los estudiantes, es decir, tiene que ser moralmente superior para poder ejercer la autoridad. En este sentido, se entrevé la exigencia de un docente perfecto, ordenado, claro, con una buena reputación, debido a la creencia social de que esto pudiese afectar de manera directa el comportamiento de los estudiantes y su autoridad ante estos.

Sánchez (2010) utiliza la metáfora del profesor como actor para explicar la manera como debe comportarse dentro del contexto educativo, al ingresar al aula se debe desprender de cualquier acervo de emociones y preocupaciones, y se convierte en un sujeto análogo de sí mismo, que actúa bajo los parámetros establecidos y aceptados en el ámbito en el que se desenvuelve, con el objetivo de captar la atención de los estudiantes y facilitar su proceso de aprendizaje.

De esta manera, se puede ver una perspectiva general de la situación de los maestros en el momento histórico que se está atravesando, en el cual se enfrenta a unos juicios sociales, que cuestionan su valía desde su práctica pedagógica, su saber y su manera de ser y estar en el mundo. Sin embargo, Domínguez (2011) manifiesta que el maestro ha sido un actor fundamental, ya que

el estudio y comprensión de su persona, la dimensión personal y subjetiva de su existir han sido soslayados en el ámbito de la educación formal, a pesar de que la docencia es una profesión fuertemente matizada por los aspectos personales y subjetivos. (p. 27)..

Considerando los retos que en la actualidad tiene el maestro en relación con su ser emocional, es importante reconocer los aspectos que matizan la docencia y comprender la manera en que la subjetividad magisterial ha sido desplazada del debate educativo. Por tal razón, se suele tener en cuenta los aspectos personales ligados a los estudiantes desde su esfera privada, aunque son escasas la reflexiones en torno al maestro desde su singularidad, y se desconoce que es un ser crítico que se encuentra inmerso en un contexto sociocultural, donde establece relaciones con sus pares y con otros actores de la comunidad educativa, de las que construye diversas narraciones.

Así mismo, el maestro es un ser único, singular, con diversas particularidades que dependen de la construcción de su subjetividad, y al estar en relación continua con los otros 
se supone abierto a la realidad y a que le permee como experiencia. La cual, depende de su esencia y le confiere un carácter, dado que construye "su realidad, sus modelos o los "lentes" desde y con los cuales examina los acontecimientos personales y profesionales entretejidos; de este modo, va configurando su realidad interna" (Domínguez, 2011, p. 27).

Al respecto, el pensamiento y la reflexión sobre la experiencia constituyen una parte fundamental del acto pedagógico, pues así el maestro evalúa sus prácticas y recupera la historicidad de la pedagogía que le ha sido arrebatada; además, se recupera la subjetividad que le ha sido sustraída al acto educativo al preguntarse por la forma en que las emociones movilizan al maestro y a sus estudiantes, pues el conocimiento está implicado de manera estrecha con estas, a pesar de la intención continua por invisibilizarlas. Al respecto, Van Manen (2016) expresa que "la noción de reflexión está implícita en el significado de la pedagogía, que, por definición, significa que la enseñanza es hecha en una forma intencional" (p. 50).

Esta comprensión no solo sucede desde la reflexión sobre la pertinencia de los contenidos, sino desde las emociones que rodean el acto educativo; acá se encuentra que comúnmente, las emociones catalogadas como poco deseables en los maestros, suelen ser las que mayor aparición hacen en el proceso de enseñanza y aprendizaje.

No obstante, en la actualidad hay una exigencia del medio sobre el maestro, que le demanda ser una persona afectiva, dejar de lado la antigua rigurosidad en su actuar y manera de relacionarse con los estudiantes, donde se caracterizaba por ser un sujeto autoritario y distante, para convertir la afectividad en una característica importante en los vínculo que se tejen dentro de la educación.

Anteriormente, el maestro era visto como una figura de autoridad, cuyo poder se basaba en la represión y en la falta de expresión de sus emociones, pues se consideraba que la expresión emocional hacia los alumnos era sinónimo de debilidad y de pérdida de poder. Actualmente, el maestro se encuentra abocado hacia un nuevo reto: ya no se pretende que sea frío e inexpresivo emocionalmente, sino que, al contrario, debe ser afectuoso. Su relación con los estudiantes debe basarse en el conocimiento de sus necesidades, particularidades y su cuidado. 
Por lo anterior, se propende por un maestro que exprese afecto aunque no sea su verdadero sentir. El maestro no solamente se debe reconocer por su aptitud de acuerdo con el título que le certifica para desempeñar la docencia, sino también, como lo describe Abramowski (2010):

la 'cuestión afectiva' y el lenguaje sentimental están adquiriendo cada vez mayor centralidad en la descripción de lo escolar, de los maestros y alumnos, y se remite a ella tanto a la hora de identificar problemas como de pensar soluciones. (p. 20).

\section{Las emociones en la experiencia del maestro}

La concepción que se ha tenido sobre las emociones ha cambiado de acuerdo con el momento histórico en el que se vive, en principio eran nombradas como pasiones, que en palabras de Abramowski (2010) "son significaciones culturales, que están inscritas en relaciones sociales y que sus sentidos se definen dentro de determinados parámetros conceptuales y valorativos" (p. 34). Según la perspectiva estoica, en la que se le daba mayor protagonismo a la razón, las emociones adquieren sentidos perjudiciales, y se convierten en aquello que le hace daño al sujeto para vivir en sociedad. Esta corriente filosófica fundada por Zenón de Citio, puso

un énfasis preponderante en nuestra forma de enjuiciar el mundo o de valorarlo al tiempo que era percibido. Ya que no podemos ser dueños de la mera percepción de la realidad que, en principio, se nos impone y no controlamos, sí lo somos, en cambio, del juicio que acompañaba a tal percepción. Habrá que aprender, pues, a transformar los juicios inconvenientes. (...) De acuerdo con tal teoría, las pasiones son 'errores del juicio' o 'conmociones del alma', desviadas de la recta razón de la naturaleza. (Camps, 2011, p. 37).

Desde esta perspectiva, las emociones son negativas, ya que no permiten el libre desarrollo del alma, especialmente con situaciones imposibles de evitar como la muerte o el dolor, por lo cual, los estoicos consideraban que era necesario aprender a controlarlas para sobrevivir en el mundo de seres vulnerables, es decir, modificar el juicio y ver de otra forma las cosas, que dejen de perturbar, con el objetivo de conseguir la ataraxia: un estado de plenitud reservado para los verdaderos sabios (Camps, 2011). 
Es por lo anterior, que al ser vistas desde una perspectiva racional se clasificaban como pasiones calientes, que deforman la realidad y pueden conducir al ser humano a cometer errores, ya que las emociones revelan su naturaleza vulnerable, explicitan la forma que tiene de ver el mundo y las diversas sensibilidades que este tiene hacia ciertos fenómenos (Camps, 2011), por ello, eran confinadas a la esfera privada, pues tenían muy pocos atributos que les permitieran ser racionales, así, eran valoradas como fenómenos que se debían aprender a manejar, pues afectaban directamente la interacción del "yo" con los "otros".

De este modo, las pasiones calientes se asocian directamente con el yo, se conforman a partir del carácter y la personalidad del sujeto, lo que entraba en contravía a lo esperado y aceptado en la vida social por la supremacía de la razón, y por ende, las pasiones 'frías' priman por encima de la subjetividad, debido a que se reflejan en una actuación prudente y provista de reflexión. Es por ello, que socialmente se buscó el traslado de las pasiones 'calientes' a la vida privada (el hogar) donde se le da un lugar de importancia y se configura la relación domesticidad-emociones (Abramowski, 2010).

Pese a este desplazamiento, la escuela no se desliga de estas pasiones, ya que allí es donde se ponen en juego las emociones de los maestros en el contacto con los estudiantes y los demás aspectos que allí convergen, sin embargo, para la sociedad, las emociones de los maestros deben ser "inteligibles, pensables, decibles y disponibles para ser sentidas en determinados tiempos y espacios" (Abramowski, 2010, p. 53).

El ámbito educativo, sin lugar a dudas, es uno de los que más ha sido impactado por transformaciones, reflexiones, propuestas, paradigmas y reformas que entran en vigencia continuamente, y le han permitido desarrollar grandes avances. Es así, como los maestros están inundados de innovaciones y políticas en esta materia, aunque, a pesar de ser quienes las llevan a cabo, se ha puesto insuficiente atención al análisis de las emocionalidades que asume y cuál es su identidad a la luz de los nuevos enfoques y exigencias educativas.

Según Van Manen (2016, p. 195), "el carácter es la identidad distintiva que marca al individuo", en la visión antropológica y filosófica de la persona, la identidad constituye su núcleo más profundo y comprende el conjunto de rasgos y cualidades 
que reconoce como propios, entendidas como las "potencias físicas y psíquicas" con las que cuenta para desarrollarse y crecer (Domínguez, 2011, p. 62). Es por ello, que lo emocional no solo emerge en situaciones educativas de riesgo, sino que también forma parte de las acciones cotidianas, del ser y de la identidad del maestro como individuo.

Tal como lo señala Camps (2011), "hay emociones que nos incitan a actuar, otras nos llevan a escondernos o a huir de la realidad. Todas las emociones pueden ser útiles y contribuir al bienestar de la persona que las experimenta, para lo cual hay que conocerlas" (p. 13). Por esto, las personas sienten y expresan sus emociones de acuerdo con unos cánones socialmente aprendidos, lo cuales varían a partir del espacio y el tiempo, y además, tienen un objeto, ya que según Marín y Saldarriaga (2019)

las emociones aparecen en relación con algo, en especial con la experiencia humana, como lo son el sentir amor, miedo, enojo o asco por el otro; (...) tienen un carácter intencional, esto es, que las emociones no hacen su aparición en relación con los sujetos en sí mismos, sino a partir de las percepciones, interpretaciones, valoraciones y los juicios que se tienen sobre alguno o algunos de sus rasgos definitorios; por lo tanto, siempre van a tener que ver con algo relacionado con alguien. Además, las emociones encarnan creencias en relación con ese alguien o con alguno de sus rasgos característicos. (pp. 37-38).

Así mismo, las emociones comportan atributos, puesto que obedecen a creencias relacionadas con las percepciones subjetivas y culturales, y se encuentran ligadas a juicios y valoraciones que las personas hacen sobre algo o alguien, de ahí que encierren un carácter de intencionalidad. Todas las emociones tienen una utilidad específica, que le permite al ser humano reaccionar ante situaciones determinadas, como es el caso de aquellas que serán desarrolladas a continuación.

\section{La empatía: más allá de ser una emoción}

Una disposición que atraviesa al ser y el quehacer del maestro es la empatía, esta, más allá de ser una emoción, es definida de manera amplia por Decety \& Jackson (2004) como una experiencia subjetiva natural de similitud entre los sentimientos expresados por sí mismo y otros, sin perder de vista a quién pertenecen. La empatía involucra, no solo la 
experiencia afectiva del estado emocional real o inferido de la otra persona, sino también un mínimo reconocimiento y entendimiento del estado emocional del otro, utilizada por los teóricos de la estética para referirse a la capacidad de percibir la experiencia subjetiva de otra persona, sin que implique necesariamente que uno actuará o se verá impelido a actuar en una forma simpática o de apoyo (Decety \& Jackson, 2004).

La empatía es indispensable para el maestro porque debe leer en los rostros del otro sus emociones para actuar en concordancia con estas, humanizar el acto educativo al reconocer al otro como un ser emocional, lo que se ha vuelto fundamental para la educación actual, de manera que le permite al maestro cumplir con la nueva exigencia moderna propuesta por Abramowski (2010), de establecer relaciones con sus estudiantes basadas en el afecto.

Esta predisposición a la comprensión del mundo emocional del otro tiene una naturaleza neutral, es decir, no es buena o mala en sí misma, pues "es una clase de actitud imaginativa y participativa" (Nussbaum, 2001, p. 321), y como tal, es una posible preparación para la acción, la cual depende del interés que se tenga respecto a la emoción que está siendo percibida, es decir, se puede ser empático y aprovecharlo para fines desprovistos de bondad (el caso del bullying) o para fines bondadosos y caritativos (la ayuda brindada a un estudiante que sufre). Por este motivo, las personas empáticas suelen sintonizar con las señales sociales sutiles que indican qué necesitan o qué requieren los demás.

Por tanto, la conciencia de sí mismo es la facultad sobre la que se erige la empatía, puesto que, cuanto más abierto esté el individuo a sus propias emociones, mayor será la destreza en la comprensión de los demás. Van Manen (2016) también aborda esta idea en su análisis del tacto pedagógico, señala que gran parte de la información empática, al ser un puente articulador entre el maestro y las emociones propias y ajenas, se relaciona íntimamente con las habilidades sociales y comunicativas, mediante las cuales el maestro expresa emociones que los estudiantes reciben y codifican, y así genera un tono de empatía mutua, por tanto, al docente le corresponde en primer lugar ser empático consigo mismo, privilegiar su autoconocimiento y tener en cuenta su sentir. 
Desde otra perspectiva, Nussbaum (2001) plantea que la empatía "a menudo acompaña la compasión, pero no es fundamental para que una persona sienta compasión; las emociones contienen elementos no cognitivos que son las sensaciones y los estados físicos del cuerpo" (p. 483). Por tanto, puede generar una ambivalencia del maestro entre la satisfacción o el malestar, la cual es descrita por maestros en diálogos informales cuando expresan,

en mi práctica sí alcanzo a sentir los dos lados: algunas veces desánimo, otras veces frustración de no saber qué hacer, cómo enfrentarme a los desaciertos de los muchachos (...); en otras ocasiones me sentía muy bien, con empatía con los niños, con una conexión muy chévere, cuando veo que aprenden y están contentos (Conversación informal con maestro, 2019).

La realidad para el maestro es que pasa en un instante de sentir emociones que lo reconfortan a emociones que lo devastan; la alegría que le genera la relación con sus estudiantes se puede convertir al minuto siguiente en insatisfacción, miedo o ira.

\section{La ira: una emoción eudemonista}

Tal como señala Nussbaum (2018), la ira encierra un contenido cognitivo intencionado que integra múltiples valoraciones, y

las estimaciones y las creencias que se relacionan con la ira son lo que [llama] eudemonistas: se construyen desde la perspectiva del agente y registran la visión personal de lo que para este importa en la vida y no desde una tabla de valores objetiva o impersonal. (p. 38).

Reconociendo la ira como una construcción social, es posible entenderla como una emoción básica y universal: básica porque involucra la supervivencia a partir de tres funciones: la facilitación del desarrollo rápido de conductas de defensa, la vigorización de la conducta y la regulación de la interacción social, y es universal, porque es inherente a la experiencia humana.

Algunos desencadenantes de esta emoción son las situaciones frustrantes y adversas, que se presentan de manera recurrente en el aula, a las cuales el maestro se enfrenta día a día y que pueden ser nombradas fácilmente en sus conversaciones cotidianas. Las primeras, se relacionan especialmente con 
situaciones de transgresión de las normas y derechos por parte de algún miembro de la comunidad educativa. Las segundas situaciones están relacionadas con experiencias desagradables, como el ruido constante, el excesivo número de estudiantes, el hacinamiento, entre otras.

En otras palabras, la ira se desencadena por una situación sobre la cual se emite un juicio al creer que este va en contravía de los intereses propios. En este mismo sentido Nussbaum (2001) asevera que para sentir ira

es necesario poseer un conjunto de creencias: que se ha infligido un perjuicio, a mí o a algo o alguien cercano a mí; que no se trata de un daño trivial, sino relevante; que fue realizado por alguien; probablemente, que fue un acto voluntario. (p. 51).

A pesar del estigma social creado bajo perspectivas de inhibición emocional que llevan a afirmar que la ira es una emoción destructiva, esta es "una parte valiosa de la vida moral, fundamental para las relaciones humanas, tanto éticas como políticas" (Nussbaum, 2018, p. 35), ya que muestra aquellas cosas que revisten importancia para el sujeto y que según su escala de valores, juicios, creencias y valoraciones, vale la pena defender.

Se comporta, a su vez, como un indicador para el sujeto y quienes lo rodean, de que se ha cometido una falta contra aquello que juzga como valioso o considera parte de su bienestar integral, es una fuente de motivaciones para abordar esta falta y funciona como disuasión para los otros, pues desalienta su agresión (Nussbaum, 2018).

\section{La vergüenza: vulnerabilidad del ser humano}

Al igual que la repugnancia, para Nussbaum (2006) la vergüenza es una emoción que se origina en la vida social, por medio de la cual se rechaza la noción de anormalidad y las debilidades del ser humano, que va más allá de una respuesta fisiológica ya que,

sirve como una manera altamente volátil con la que los seres humanos negocian algunas tensiones inherentes a su humanidad, es decir, en su conciencia de sí mismos como seres humanos finitos y marcados por demandas y expectativas exorbitantes. (p .206). 
A lo largo de la historia, la vergüenza ha estado vinculada con la humillación, la cual pone de manifiesto la vulnerabilidad del ser. Además ha sido utilizada para condenar, manipular o poner en ridículo a las personas. Nussbaum (2006) retoma los planteamientos de Aristófanes, quien la describe como una emoción dolorosa, basada en reconocer la falta de omnipotencia y de control por parte de los seres humanos, lo que deja de lado la sensación de estas facultades, sentimientos o emociones que se encuentran encadenados a la memoria primitiva de los sujetos, por lo cual aduce que

intuimos que debemos ser completos y quizá lo fuimos en algún momento, y sabemos que ya no lo somos. (...) Parece plausible que Aristófanes tenga razón: una clase de vergüenza primitiva por el hecho mismo de ser humanos y no completos subyace a los tipos más específicos de vergüenza que sentimos más tarde respecto de limitaciones e inadecuaciones. (...) Porque la vergüenza involucra el hecho de advertir que se es débil e inadecuado en algunos sentidos en los que se espera ser adecuado. La reacción consiste en esconderse de la mirada de quienes verán la deficiencia, en cubrirla. (p. 217).

Al estar relacionada con la vulnerabilidad, la vergüenza se puede apreciar como una emoción dolorosa, lo cual responde de alguna manera a no poder lograr el ideal de perfección. Esta aparece justo después del nacimiento como uno de los afectos primarios, y surge de manera progresiva desde los primeros años de vida: requiere pensamiento, un sentido embrionario del propio ser, lo que no significa que el sujeto deba tener baja autoestima.

Lo anterior hace que existan diferentes tipos de vergüenzas, una de ellas es la vergüenza primitiva, la cual se encuentra relacionada con la "omnipotencia infantil y con el fracaso narcisista". En este sentido, la vergüenza se constituye "en una conciencia de inadecuación que precede cualquier aprendizaje particular de normas sociales, aunque en la vida posterior se verá sesgada por el aprendizaje social" (Nussbaum, 2006, p. 219).

De esta manera, la vergüenza y el narcisismo se validan el uno al otro, dado que la primera forma de experimentación del sujeto es consigo mismo, además, dicha emoción se encuentra vinculada con el bochorno, el cual, a pesar de que se ha comprendido como humillación es diferente, ya que la humillación es una forma seria de ser avergonzado mientras 
que el bochorno es de menor intensidad que la vergüenza y depende de un estado emocional subjetivo (Nussbaum, 2006).

Esta emoción hace parte de la cotidianidad del maestro, la cual le hace ser consciente de algunas adecuaciones que tiene respecto a normas sociales establecidas para él, pues al ser sujeto de idealización, está sometido a niveles más altos de exigencia y crítica. La experimenta principalmente al ser confrontado en su realidad contra los estereotipos que rodean su figura magisterial: situaciones como haber cometido alguna injusticia contra un estudiante, recibir un trato inadecuado por parte de un padre de familia o directivo, ser despojado del orgullo que tradicionalmente tenía la docencia, ser cuestionado por la comunidad educativa, entre otras, confrontan al maestro por medio de la vergüenza y la humillación y lo mueve a la autoevaluación de su identidad, obligándole a repensar su forma de ser maestro y a corregirla de acuerdo con las demandas que se le realizan, como forma de evitar ser expulsado del círculo social en que se desenvuelve.

\section{La repugnancia: mecanismo de protección ante la contaminación}

La repugnancia es concebida como una de las primeras emociones que experimenta el ser humano, la cual se va desarrollando durante la primera infancia, a medida que los padres le enseñan a sus hijos a cuidar (ocultar, limpiar) su cuerpo como medio de protección y de autoconservación, y se asocia con la idea de evolución: se debe proteger de la contaminación (desechos o fluidos corporales: heces, saliva, semen, etc.) que pone en riesgo su humanidad y lo acerca a su condición animal.

Esto se evidencia en algunas situaciones en las cuales el maestro se ve enfrentado a los fluidos corporales y los olores de sus estudiantes. Esta emoción aparece de forma preponderante, puede llegar a afectar fuertemente el cumplimiento de la demanda moderna del afecto magisterial (Abramowski, 2010) y ser considerada como rechazo y discriminación.

Esta emoción también se relaciona con las normas establecidas por una sociedad o lo que cataloga como bueno, malo, permitido y prohibido. Al respecto, Kass (1998, citado por 
Nussbaum, 2001) asemeja la repugnancia a la inteligencia, o "sabiduría", ya que alerta al sujeto para no trasgredir normas, además, menciona que se puede asociar con la moral, por lo cual se puede utilizar como una especie de juicio de valor sobre situaciones, personas u objetos.

Abramowski (2010) expresa que "en el abanico de los afectos difícilmente enunciables y sentibles está el propio rechazo hacia determinados alumnos" (p. 59), este puede ser generado por ciertas formas de comportarse que el maestro concibe como intolerables y le suscitan esta emoción, es decir, es una forma de repugnancia asociada con la idealización, y aparece la razonabilidad y la irracionalidad como justificación.

Las emociones siempre tienen un contenido cognitivo, lo que se relaciona con el término "moral compartida" (Nussbaum, 2006), que hace alusión a los valores compartidos socialmente, en los que la repugnancia es una de las fuerzas que respaldan la ley moral. Se puede decir, que el sujeto maestro se encuentra cobijado por el manto de la moral compartida, desde la cual se le hacen demandas a su manera de emocionarse, pues la emoción va ligada a la acción, y le lleva a reaccionar mediante muestras de rechazo y repugnancia ante actitudes de su estudiantado que van en contra de lo establecido y aceptado socialmente, como la discriminación, la intolerancia y la agresión.

Desde este punto, la repugnancia se comprende como una emoción aprendida por medio de las interacciones sociales, ya sea en la familia o en el contexto en el que se desenvuelve el sujeto y se configura en un mecanismo de presión para aquellos individuos que se desvían del orden establecido, para que, evitando perder su núcleo social, redireccione su actuación a lo que la sociedad ve como bueno y permitido. Es por ello que para Nussbaum (2006) "la repugnancia es un producto cultural y, por lo tanto, un buen índice de lo que nos ha llegado a importar socialmente" (p. 101).

\section{El miedo:}

\section{una emoción conservadora del bienestar humano}

Es notable observar que emociones como la repugnancia y la vergüenza vienen de la mano con el miedo, el cual al ser una emoción básica, se convierte en indispensable para la 
vida del ser humano, ya que por esta se vislumbran diversas formas de comprender el mundo que lo afectan directa o indirectamente, por lo que su comprensión es fundamental para entender la forma en que se tejen las relaciones sociales.

El miedo es abordado por Camps (2011) como una emoción protectora, se sustenta en creencias que le generan al hombre incertidumbre al estar de cara al futuro, y le otorga responsabilidad hacia este. Así mismo, esta emoción busca evitar el mal, lo ve como algo destructivo y penoso, e inclina al ser humano a actuar hacia la paz. Según Gordon, citado por Camps (2011), el miedo se divide en dos tipos: epistémico y fáctico. El miedo epistémico se basa en el miedo al futuro, mientras el miedo fáctico está relacionado con el pesar y mira al pasado.

Por esto se puede entender el miedo como una emoción conservadora del bienestar humano y la paz común, que le brinda al hombre la responsabilidad de actuar frente al mal probable y asumir una responsabilidad moral y ética. Adicionalmente, puede verse como el sentimiento de ser susceptible al peligro, el cual es reciclado culturalmente de experiencias pasadas, Ileva al sujeto a reconocer su propia vulnerabilidad.

Esta emoción se relaciona fuertemente con la incertidumbre, la cual lleva implícita la ignorancia respecto a la amenaza hacia las personas, el orden social, la supervivencia y el lugar de ser en su entorno. El mundo educativo está Ileno de incertidumbre, y esto se evidencia de forma constante en los sucesos del aula, el miedo hace parte esencial de las emociones que afectan al maestro, pues debe prever lo que pueda suceder consigo mismo y sus educandos, ya que al tener un rol de responsabilidad, debe velar por protección y seguridad para sí mismo y aquellos que se encuentran bajo su cargo.

El miedo no solo se da por la responsabilidad otorgada, sino que también se relaciona con los estereotipos y creencias en torno al ser magisterial, que llega a sentir temor por situaciones como: ver afectada su imagen ante la comunidad educativa, marcar de forma negativa la vida de algún estudiante, tener repercusiones legales ante algún suceso ocurrido en el aula, a las amenazas de los padres de familia, actores armados y directivos que puedan poner en riesgo 
su puesto de trabajo y su profesión. Lo anterior muestra su carácter protector al motivar a evitar el mal (Camps, 2011).

\section{Conclusiones}

La empatía (entendida como disposición para sentir emociones), la ira, la repugnancia, la vergüenza y el miedo, hacen parte de la cotidianidad del ser maestro y se convierten en emociones abrumadoras, debido a la idealización e instrumentalización al cual ha sido sometido, cotidiana e históricamente, y dificulta su expresión emocional en los espacios educativos, lo cual incide directamente en su práctica pedagógica, y en su manera de pertenecer a la institución educativa.

Por esta razón, brindarle al maestro la oportunidad de comprender y expresar su subjetividad se convierte en un reto esencial para la pedagogía actualmente, pues solo por medio de su reconocimiento como ser integral, se puede cambiar el discurso de control emocional que se evidencia en torno a la educación y permitir que este pueda empoderarse y recuperar el carácter subjetivo y crítico de la enseñanza.

En este sentido, las prácticas afectivas, al igual que otras dinámicas que tienen lugar en el contexto educativo, se van construyendo en su ejercicio profesional, así, se establece una forma de vivenciar y expresar sus emociones. Para Abramowski (2010), "hay un qué, cómo, cuándo, dónde afectivo que auxilia a los maestros a formatear sus afectos, a apelar a determinadas emociones en determinados momentos, dejando a un lado otras. Los maestros, en tanto tales, no sienten 'cualquier cosa'" (p. 54), o no le es permitido en muchas ocasiones sentirlas.

\section{Referencias}

Abramowski, A. (2010). Maneras de querer: Ios afectos docentes en las relaciones pedagógicas. Buenos Aires: Paidós.

Camilloni, A. (2011). La formación docente como política pública: consideraciones y debates, Mar de la Plata: Revista de Educación 3(2), 11-28. 
Camps, V. (2011). El gobierno de las emociones. Barcelona: Herder.

Decety, J. \& Jackson, P. (2004). The functional architecture of human empathy. Behavioral and cognitive neuroscience reviews, 3(2), 71-100. doi: 10.1177/1534582304267187.

Díaz, M. (1995). Aproximaciones al campo intelectual de la educación. Escuela, poder y subjetividades. Madrid: La Piqueta.

Domínguez, C. (2011). El maestro como persona y sus historias de docencia. (2), 25-45. Chihuahua: StudyLib. https://studylib.es/doc/6587269/el-maestro-como-personay-sus-historias-de-docencia

Marín, M., y Saldarriaga, J. (2019). Emociones políticas: un abordaje epistemológico. Emociones políticas en niños y niñas. Medellín: Editorial Universidad de Antioquía.

Nussbaum, M. (2006). El ocultamiento de lo humano. Repugnancia, vergüenza y ley. Buenos Aires: Katz Editores.

Nussbaum, M. (2018). La ira y el perdón: resentimiento, generosidad, justicia. México: Fondo de cultura Económica.

Nussbaum, M. (2001). Paisajes del pensamiento: la inteligencia de las emociones. Barcelona: Paidós.

Sánchez, J. (2008) El profesor o Morfeo. El profesor en la trinchera. 97-114. Madrid: La Esfera.

Van Manen, M. (2016). Pedagogical tact. New York: Tayler \& Francis.

Varela, J.(1991): El triunfo de las pedagogías psicológicas. (198) 56-59. Logroño: Cuadernos de Pedagogía.

Zuluaga, O. (1999). Pedagogía es historia: la historicidad de la pedagogía, la enseñanza, un saber de saber. Bogotá: Anthropos. 